\title{
Fluoroscopic Intraoperative Images Produce Higher Image Quality and Decrease Total Radiation Exposure Compared to Radiographic X-ray Images in Patients After Primary Total Knee Arthroplasty
}

\author{
TILMAN GRAULICH ${ }^{1}$, JULIUS GERHARDY ${ }^{1}$, MARCUS ÖRGEL ${ }^{1}$, TAREK OMAR-PACHA ${ }^{1}$, \\ MOHAMED OMAR $^{1}$, CHRISTIAN KRETTEK $^{1}$ and DANIEL GUENTHER ${ }^{2}$ \\ ${ }^{1}$ Trauma Department, Hannover Medical School, Hannover, Germany; \\ ${ }^{2}$ Department of Orthopaedic Surgery, Trauma Surgery, and Sports Medicine, \\ Cologne Merheim Medical Centre, Witten/Herdecke University, Cologne, Germany
}

\begin{abstract}
Background/Aim: Proper radiographic documentation of implant alignment is needed to analyse malrotation and malpositioning. We examined whether intraoperative fluoroscopic images can achieve more accurate image quality than postoperative radiographic $X$ ray images. Patients and Methods: We prospectively analysed 30 consecutive patients after total knee arthroplasty (TKA). We compared intraoperative fluoroscopic images with postoperative radiographic $X$-ray images. Radiation exposure was documented. Results: Fluoroscopic anteriorposterior images could achieve accurate image quality in $77 \%$ compared to $60 \%$ in radiographic images $(p=0.016)$ and $54 \%$ compared to $34 \%$ on lateral view, respectively $(p=0.008)$. Very good intra-observer correlation for fluoroscopic images could be achieved for femoral $\alpha$ angle with 0.84. Radiation exposure was 0.087+/-0.128 mGy. Conclusion: We observed significantly better image quality in fluoroscopic images than in radiographic X-ray images. The observed radiation exposure is lower than those expected for radiographic X-ray images. We conclude that fluoroscopic images can produce higher image quality and decreased radiation exposure.
\end{abstract}

This article is freely accessible online.

Correspondence to: Tilman Graulich, MD, Trauma Department, Hannover Medical School, Carl-Neuberg-Straße 1, 30625 Hannover, Germany. Tel: +49 17615326723, Fax: +49 511532 5877, e-mail: graulich.tilman@mh-hannover.de

Key Words: Total knee arthroplasty, fluoroscopy, radiographic images, X-ray, accuracy, precision.
Exact component fixation and alignment of total knee arthroplasty (TKA) is imperative to reduce postoperative pain and obtain patient satisfaction and good clinical function (1-4). Therefore proper radiographic documentation of coronal and sagittal implant alignment is needed to analyse malrotation and malpositioning $(5,6)$. Due to a lack of consistency in roentgenographic reporting of findings, rating systems have been described $(2,5,7)$. The original Knee Society Clinical Rating System had been described in 1989 by Ewald et al. and has been adapted by Meneghini et al. They intended to modernized and updated the radiographic evaluation system as a rising diversity and complexity in implant design came up. Therfore they aimed to accumulate radiographic data in a standardized manner (5, $7,8)$. In addition, a well aligned postoperative image is inevitable for compareability to detect implant loosening over time.

However, accurate and precise radiographic images are difficult to obtain as they depend on several parameters. Using either standing or portable radiographs for the measurement of the knee joint centre gives significant different values due to changes in the rotation of the knee in the presence of knee flexion (9). Limb rotation and knee flexion of $10^{\circ}$, either alone or in combination, has a significant effect on measured values of the anatomic alignment (10-13). Further patients' specific parameters like pain and casts can make proper radiograph acquisition challenging.

Looking for improvement of measured parameter reliability and reproducibility, the use of 3D-CT has shown better reliability than $2 \mathrm{D}-\mathrm{CT}$. However, radiation exposure is much higher compared to radiographic X-ray images $(1,14)$.

The use of intraoperative fluoroscopy of the anaesthetized patient reduces the patient's incompliances due to pain and 
could be a tool to improve image acquisition. It has been shown that fluoroscopically assisted radiographs increased the sensitivity of detecting tibial component loosening over standard radiographic X-rays after TKA during regular follow up (15). The amount of additional radiation dose is variable between the patients as the number of images and the exposure time varies. An extra amount corresponding to 8-12 images is needed to produce accurate fluoroscopic images. This summarizes about 5-10 seconds of fluoroscopic exposure time. An fluoroscopic exposure rate of 1 $\mathrm{mGy} /$ minute is described in the literature with a consecutive exposure of 0.05-0.15 mGy (15). In comparison, a standard knee radiographic image has an exposure of 0.05-0.1 mGy as well. Taking the specific tissue properties into consideration, the effective dose is lower than $0.005 \mathrm{mSv}$ per standard knee radiograph (15).

The purpose of this study was to analyse if i) intraoperative fluoroscopy can produce a more accurate image quality than postoperative X-ray and ii) differences in the TKA-analysing angles, as described by the Knee Society Clinical Rating System, can be observed.

The hypothesis of the study was that fluoroscopic images can produce more accurate images than postoperative radiographic $\mathrm{X}$-ray and that measured angles are different between fluoroscopic images and postoperative X-rays.

\section{Patients and Methods}

We prospectively analysed 28 consecutive knees in 30 patients who underwent TKA in our level one trauma centre. Two patients had to be excluded due to incomplete image acquisition with missing radiographic $\mathrm{X}$-ray patellar images due to pain during flexion. The sample size was restricted due to the prospective design of the study, as well as radiation exposure of the patients. The knee implant used in all cases was the iTotal- Conformis knee prosthesis eather cruciate retaining (CR) or posterior stabalized (PS) (ConforMIS Inc., Billerica, MA, USA). The study was approved by our local ethics committee (Nr 7729_BO_S_2018). Informed consent was obtained from the patients.

Fluoroscopy- and radiographic X-ray imaging. Intraoperative fluoroscopic images were taken by a board certified orthopaedic surgeon. For the anterior-posterior (ap) view, the patient was in supine position, and the patellar was determined by palpation. The $\mathrm{X}$-ray beam was set perpendicular to the joint line and perpendicular to the midshaft of the femur and tibia (7). For the lateral view, the $\mathrm{X}$-ray beam was directed laterally, again perpendicular to the joint line. The patella was evaluated using $30^{\circ}$ axial-/skyline-/Merchantview $(7,8)$. The images were repeated until the surgeon believed to have accurate image quality. Imaging was considered accurate if i) central positioning of the patella in the AP view and $1 / 3$ of the fibular head was behind the tibia, ii) complete overlapping of the medial and lateral condyle in the lateral view and iii) the patella was observed in the trochlear groove $(8,16)$.

Postoperatively, radiographic X-ray images were taken by trained radiological medical techniqual assistants (MTA). For the ap view, the X-ray beam was perpendicular to the joint line while for the lateral view, the beam was directed laterally and perpendicular to the joint line and for the patella we used $30^{\circ}$ axial views. Images were not repeated if they were not strictly correct. Both, fluoroscopic images and radiographic X-ray images were evaluated by two trained orthopaedic surgeons as either accurate or nonaccurate (yes/no) twice in an interval of four weeks.

Measurements using the modern knee society radiographic evaluation system.

Anteriorposterior view/Coronal alignment: The $\alpha$ angle for alignment of the femoral component was measured between the femoral shaft axis and the articular surface of the femoral component. The $\beta$ angle for alignment of the tibial component, defined as the angle between the tibial shaft axis, which was determined by the center of four carticalices-marking dots, and the articular surface of the tibial component, was measured $(1,5,7)$ (Figure $1 \mathrm{a}, \mathrm{d}$ ).

Lateral view/Sagittal alignment: The $\gamma$ angle for alignment of the femoral component was measured between the most distal femoral fixation surface and the femoral shaft axis. The $\delta$ angle or tibial slope angle was measured between the tibial shaft axis and the articular surface line of the tibial component $(1,5,7)$ (Figure $1 \mathrm{~b}, \mathrm{e}$ ).

Merchant view/Patellofemoral alignment: The $\gamma$ angle or patellar tilt angle was determined by the anterior surface of the femoral component and the axis of the patellar $(1,5,7)$ (Figure $1 \mathrm{c}, \mathrm{f})$.

Statistical analysis. An a priori sample size calculation was performed on the given data by Chalmers et al. (15). A required sample size of 26 knees was calculated. Data was summarized using Microsoft Excel ${ }^{\circledR}$ (Microsoft Corporation, Redmond, WA, USA) software and statistical analysis was performed with IBM SPSS Statistics ${ }^{\circledR}$ Version 25 (SPSS Inc., Chicago, IL, USA). Angles on flouroscopic images and radiographic X-ray images were measured in random order. For intra-observer reliability, Pearson correlation testing was performed for scale-based variables and Spearman correlation testing for nominal based variables. To assess interobserver reliability, the intraclass correlation coefficients (ICCs) between the measurements were compared. Perfect reliability is indicated by an ICC value of 1 , very good 0.81 to 1 , good 0.61 to 0.80 , moderate 0.41 to 0.60 and poor reliability by ICC $<0.40$ (15). To evaluate the precise image quality, means between all measurements were compared. Data were tested for normal distribution with the Kolmogorov Smirnoff Test. If data were normally distributed for comparative statistics, the parametric $t$-test was used. If data were not normally distributed, the non-parametric Mann-Whitney $U$-test was used. To determine inter-observer reliability, the Pearson correlation $r$ of the two measurements was calculated. Data was described by mean \pm standard deviation defining significance level at $\alpha=0.05$.

\section{Results}

We included 28 consecutive knees in 30 patients. Out of these we included 20 men and 8 women with a mean age of $68+/-13$ (range=25-87) years. Fluoroscopic images could achieve excellent image quality on AP images in $77 \%$ 

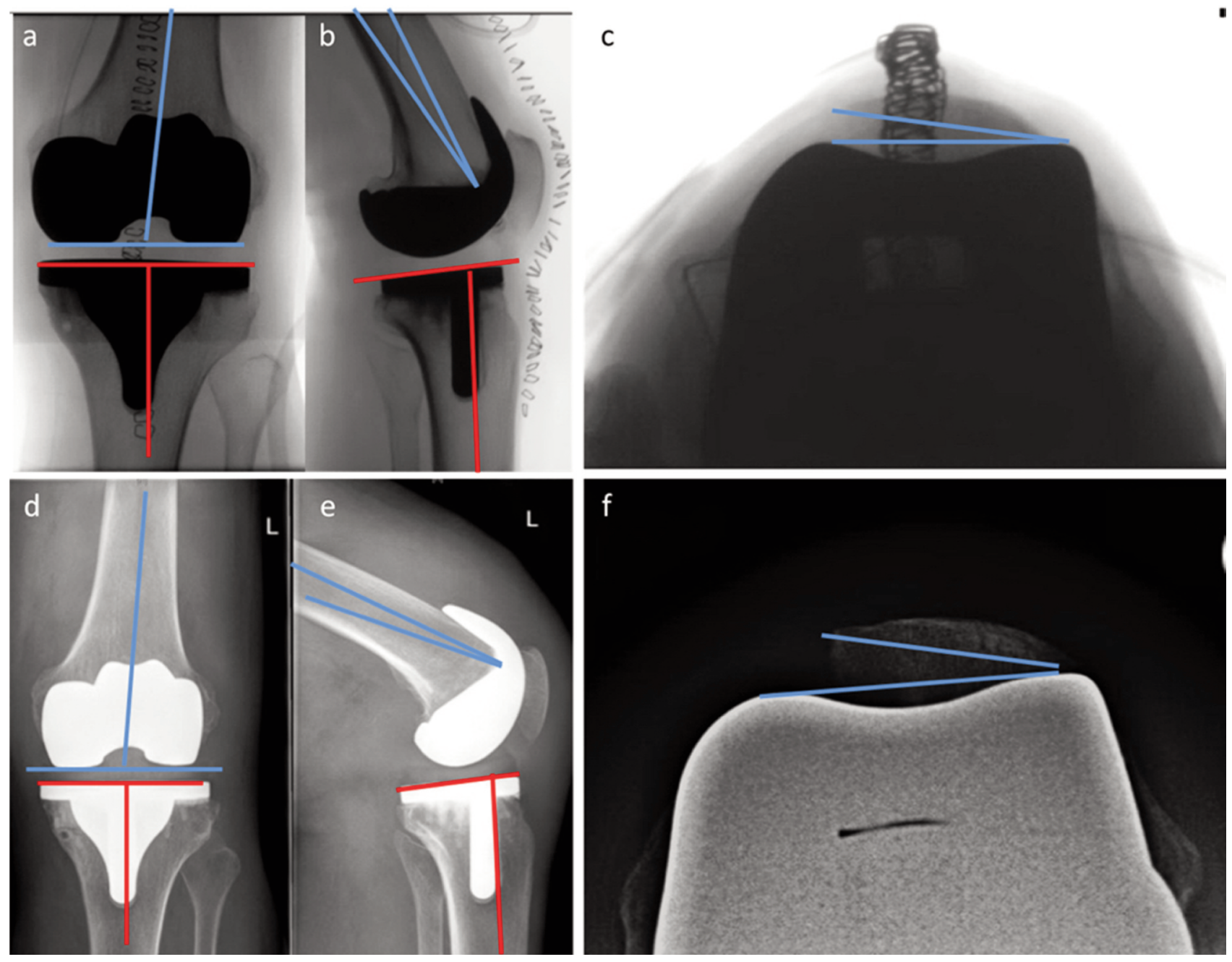

Figure 1. Flouroscopic image and radiographic X-ray image analysis and angle acquisition: a-c: flouroscopic images, d-f: radiographic X-ray images. a,d: Anteriorposterior view/Coronal alignment: BLUE. The $\alpha$ angle for alignment of the femoral component was measured between the femoral shaft axis and the articular surface of the femoral component. RED: The $\beta$ angle for alignment of the tibial component between the tibial shaft axis and the articular surface of the tibial component was measured. (1,5,7) b,e: Lateral view/Sagittal alignment. BLUE: The $\gamma$ angle for alignment of the femoral component was measured between the most distal femoral fixation surface and the femoral shaft axis. RED: The $\delta$ angle or tibial slope angle was measured between the tibial shaft axis and the articular surface line of the tibial component. $c . f:$ Merchant view/Patellofemoral alignment.: BLUE: The $\gamma$ angle or patellar tilt angle was determined by the anterior surface of the femoral component and the axis of the patellar.

compared to $60 \%$ in radiographic $\mathrm{X}$-ray images $(p=0.016)$. Concerning lateral images, fluoroscopic images showed $54 \%$ accurate image quality compared to $34 \%$ in radiographic Xray images $(p=0.008)$. On patella images, fluoroscopic images showed no difference in image quality compared to radiographic images $(40 \%$ vs. $50 \% ; p=0.217)$.

Intra-observer correlation was heterogeneous (Table I). Very good correlation could be achieved for the femoral $\alpha$ angle with 0.84 and good correlation for the patella $\gamma$ angle and femoral $\gamma$ angle with 0.773 and 0.657 , respectively, on fluoroscopic images. For correlation between fluoroscopic and radiographic X-ray images, good correlation could be achieved for femoral $\alpha$ angle with 0.692 . Further correlation testing showed only poor results.

Inter-observer correlation for fluoroscopic $v s$. fluoroscopic images was very good for femoral $\alpha$ angle with 0.844 and good for patellar $\gamma$ angle with 0.726. Fluoroscopic- $v s$. radiographic X-ray images showed only poor correlation between both observers (Table II).

Comparing fluoroscopic and radiological data, the femoral flexion angle $\alpha$ was $94^{\circ}+/-4^{\circ}$ on fluoroscopic images $v s .95^{\circ}+/-$ $3^{\circ}$ on postoperative radiographic $\mathrm{X}$-ray images $(p=0.283)$. The 
Table I. Intra-observer reliability for measured fluoroscopic and radiographic angles. Intraclass correlation coefficients (ICC). AP, Antero-posterior.

\begin{tabular}{|c|c|c|c|}
\hline & \multicolumn{3}{|c|}{$\begin{array}{c}\text { Within R1 (two measurements) } \\
\text { Reliability }\end{array}$} \\
\hline & $\begin{array}{l}\text { Mean } \\
\text { Pearson } \mathrm{r}\end{array}$ & $\begin{array}{l}\text { Spearman } \\
\text { Correlation }\end{array}$ & $p$-Value \\
\hline \multicolumn{4}{|c|}{$\begin{array}{l}\text { Fluoroscopic vs. Fluoroscopic } \\
\text { imaging }\end{array}$} \\
\hline AP-view & & 0.302 & 0.109 \\
\hline Lateral view & & 0.486 & 0.009 \\
\hline Patellar view & & 1 & $<0.001$ \\
\hline Femoral $\alpha$ angle & 0.842 & & $<0.001$ \\
\hline Tibial $\beta$ angle & 0.335 & & 0.081 \\
\hline Femoral $\gamma$ angle & 0.657 & & $<0.001$ \\
\hline Tibial $\delta$ angle & 0.369 & & 0.054 \\
\hline Patellar $\gamma$ angle & 0.773 & & $<0.001$ \\
\hline \multicolumn{4}{|c|}{$\begin{array}{l}\text { Fluoroscopic } v s . \text { Radiographic } \\
\text { imaging }\end{array}$} \\
\hline AP-view & & 0.221 & 0.259 \\
\hline Lateral view & & 0.043 & 0.828 \\
\hline Patellar view & & 0.27 & 0.164 \\
\hline Femoral $\alpha$ angle & 0.692 & & $<0.001$ \\
\hline Tibial $\beta$ angle & 0.57 & & 0.002 \\
\hline Femoral $\gamma$ angle & 0.341 & & 0.076 \\
\hline Tibial $\delta$ angle & 0.394 & & 0.038 \\
\hline Patellar $\gamma$ angle & 0.52 & & 0.005 \\
\hline
\end{tabular}

Bold values show statistical significance.

tibial angle $\beta$ was $88^{\circ}+/-2^{\circ}$ on fluoroscopic images $v s .88^{\circ}+/-$ $2^{\circ}$ on postoperative radiographic X-ray images $(p=0.681)$, the femoral flexion $\gamma$ was $13^{\circ}+/-3^{\circ}$ on fluoroscopic images $v s$. $10^{\circ}+/-2^{\circ}$ on postoperative radiographic X-ray images $(p=0.004)$, the tibial angle $\delta$ was $89^{\circ}+/-2^{\circ}$ on fluoroscopic images $v s .87^{\circ}+/-3^{\circ}$ on postoperative radiographic X-ray images $(p=0.145)$ and on patellar skyline- view the patellar tilt angle $\gamma$ was $4.5+/-8$ on fluoroscopic images and $1+/-8$ on postoperative radiographic X-ray images $(p=0.015)$.

Radiation. Radiation exposure was $0.087+/-0.128 \mathrm{mGy}$. Exposure time was $20+/-21$ s. Exposure rate was not significantly different for good/not-good AP-images, lateralimages and patella-images $(p>0.05)$.

\section{Discussion}

In our study we compared intraoperative fluoroscopic- and postoperative radiographic X-ray images of the knee after TKA for their accuracy and precision, determined intra- and inter- observer reliability, and measured radiation exposure. Three major findings could be observed. First, we could find significant more accurate image quality in fluoroscopic images than in radiographic images. Second, our data show
Table II. Inter-observer reliability for measured fluoroscopic and radiographic angles. Intraclass correlation coefficients (ICC).

\begin{tabular}{lcc}
\hline & \multicolumn{2}{c}{ R1 mean $v s$. R2 } \\
\cline { 2 - 3 } & $\begin{array}{c}\text { Fluoroscopy } v s . \\
\text { Fluoroscopy }\end{array}$ & $\begin{array}{c}\text { Fluoroscopy } v s . \\
\text { Radiographic images }\end{array}$ \\
\cline { 2 - 3 } & ICC & ICC \\
\hline Femoral $\alpha$ angle & 0.844 & 0.094 \\
Tibial $\beta$ angle & 0.332 & 0.452 \\
Femoral $\gamma$ angle & 0.65 & 0.014 \\
Tibial $\delta$ angle & 0.364 & 0.289 \\
Patellar $\gamma$ angle & 0.726 & 0.063 \\
\hline
\end{tabular}

very good intra- and inter-observer correlation for femoral $\alpha$ angle and patellar $\gamma$ angle on fluoroscopic and radiographic images. Further good intra- and inter-observer correlations could be observed. Third, the observed radiation exposure is equal to those described in the literature and lower than those expected for radiographic X-ray images. We therefore conclude that fluoroscopic intraoperative images can produce a better image quality and reduce total radiation exposure in patients after TKA. That means that the hypothesis of the study, that a better image accuracy can be achieved by fluoroscopic images was confirmed by our data. Differences in precision of the measured angles were seen in tibial angles, but not in femoral and patellar angles, which confirms our second hypothesis only partially.

Fluoroscopic images have been shown to improve detection of radiolucent lines $(17,18)$. The general benefit of fluoroscopic images compared to radiographic X-ray images to detect pathologies like loose components in TKA is an improvement of $10 \%$ precision on the AP and lateral views (15). Our data show fluoroscopic images can produce nearly $20 \%$ more accurate images in both the AP and lateral view than conventional postoperative radiographic images. Additionally, we have to consider that two patients were not able to participate in patellar imaging due to pain. This might even increase the number of accurate images. We believe that further improvements of fluoroscopic image accuracy were sacrificed in order to reduce the exposure to intraoperative radiation. We therefore think that fluoroscopic images might help to improve the analysis of malpositioning and malrotation and help to detect implant loosening $(5,6)$. This has to be tested in further analyses.

Correlation testing for fluoroscopic images $v s$. fluoroscopic images, revealed very good to good intra- and inter-observer correlation. As fluoroscopic- and radiographic X-ray images differed, poor intra- and inter-observer correlation could be observed. Interestingly tibial angles showed worse correlation testing than femoral angles. For fluoroscopic images on AP 
view, less tibial than femoral bone for reliable tibial shaft axis determination during the measurement of the $\beta$ angle might have been the reason for poor intra- and inter-observer correlation. A recent study has shown that postoperative standing long leg radiographic X-ray images $(30 \times 60 \mathrm{~cm})$ after primary TKA are an equal accurate procedure to determine both anatomical and mechanical axis compared to standing long lag radiographic X-ray images (19). As the distance of the knee to the image intensifier correlates with the magnification and the size of the projected field of view, differences in the amount of the projected tibial shaft might have been present between the images. A less precise determination of the measured angles and thus a smaller intra- and inter-rater correlation might have been the result. Furthermore, the tibial joint line to determine the $\delta$ angle/tibial slope seems to be more affected by rotation than femoral angles. Limb rotation and knee flexion of $10^{\circ}$, either alone or in combination can significantly affect measured values of the anatomic alignment; this can explain the poor correlation of measured angles between fluoroscopic images and radiographic X-ray images (10-13).

Furthermore the measured radiation exposure with $0.087+/-0.128 \mathrm{mGy}$ is comparable to data from the literature (15). Whereas one standard knee radiographic image has an exposure of $0.05-0.1 \mathrm{mGy}$, our total image acquisition radiation exposure is approximately $1 / 3$ of total postoperative radiation and we therefore believe that fluoroscopic images should be used to gain better images and improve patients' safety by means of reduced radiation exposure. We believe that, as fluoroscopic images can be more precise than radiographic X-ray images, postoperative radiographic X-ray images are not necessary anymore.

Of note, the measured angles and images were obtained from patients undergoing TKA with prothesis from one manufacturer (Confomis ITotal eather CR or PS). Therefore, the external validity to other implants is reduced, however, internal validity, comparing data undergoing the same procedure, is high. The small sample size is one limitation. The sample size was restricted due to the prospective design of the study including radiation exposure of the patients. The number of patients is comparable to the literature and significant differences could be measured. Patient number is furthermore slightly above the required sample size as determined by a priori power analysis. Fluoroscopic images and radiographic images have not been obtained by the same surgeon. However, as radiographic images have been obtained by trained MTA performing radiographic images on a daily routine base, the results are even more impressive.

\section{Conclusion}

We could find significantly better image quality in fluoroscopic images than in radiographic X-ray images. Our data show very good intra- and inter-observer correlation for the femoral $\alpha$ angle and patellar $\gamma$ angle on fluoroscopic and radiographic images. The observed radiation exposure is equal to those described in the literature and lower than those expected for radiographic X-ray images. We therefore conclude that fluoroscopic images can produce higher image quality and decrease the total radiation exposure compared to radiographic X-ray images in patients after TKA.

\section{Conflicts of Interest}

The Authors declare no conflicts of interest.

\section{Authors' Contributions}

Data acquisition: TG, JG. Drafting and revising the manuscript: TG, JG, TOP, MÖ, MO, DG. Analysis and interpretation of data: TG, CK, DG. Study conception and design: TG, CK, DG.

\section{References}

1 Hirschmann MT, Konala P, Amsler F, Iranpour F, Friederich NF and Cobb JP: The position and orientation of total knee replacement components. J Bone Joint Surg Br 93(5): 629633, 2011. PMID: 21511928. DOI: 10.1302/0301-620X.93B5. 25893

2 Elmallah RK, Scuderi GR, Jauregui JJ, Meneghini RM, Dennis DA, Backstein DB, Bourne RB, Mont MA and Knee Society Roentgenographic Workgroup: Radiographic evaluations of revision total knee arthroplasty: A plea for uniform assessments. J Arthroplasty 30(11): 1981-1984, 2015. PMID: 26364904. DOI: 10.1016/j.arth.2015.08.013

3 Brugioni DJ, Andriacchi TP and Galante JO: A functional and radiographic analysis of the total condylar knee arthroplasty. J Arthroplasty 5: 173-180, 1990. PMID: 2358816. DOI: 10.1016/ s0883-5403(06)80237-8

4 Longstaff LM, Sloan K, Stamp N, Scaddan M and Beaver R: Good alignment after total knee arthroplasty leads to faster rehabilitation and better function. J Arthroplasty 24(4): 570-578, 2009. PMID: 18534396. DOI: 10.1016/j.arth.2008.03.002

5 Meneghini RM, Mont MA, Backstein DB, Bourne RB, Dennis DA and Scuderi GR: Development of a modern knee society radiographic evaluation system and methodology for total knee arthroplasty. J Arthroplasty 30: 2311-2314, 2015. PMID: 26122112. DOI: $10.1016 /$ j.arth.2015.05.049

6 Yaffe MA, Koo SS and Stulberg SD: Radiographic and navigation measurements of TKA limb alignment do not correlate. Clin Orthop Relat Res 466: 2736-2744, 2008. PMID: 18752032. DOI: $10.1007 / \mathrm{s} 11999-008-0427-9$

7 Ewald FC: The knee society total knee arthroplasty roentgenographic evaluation and scoring system. Clin Orthop Relat Res 248: 9-12, 1989. PMID: 2805502.

8 Bach CM, Nogler M, Wimmer C, Ogon M, Steingruber IE and Peer S: Radiographic assessment in total knee arthroplasty. Clin Orthop Relat Res 385: 144-150, 2001. PMID: 11302305. DOI: 10.1097/00003086-200104000-00022

9 Younger ASE, Beachamp CP, Duncan CP and McGraw RW: Position of the knee joint after total joint arthroplasty. J 
Arthroplasty 10: 53-61, 1995. PMID: 7730831. DOI: 10.1016/ s0883-5403(05)80101-9

10 Lonner JH, Laird MT and Stuchin SA: Effect of limb rotation on radiographic alignment in total knee arthroplasties. Clin Orthop Relat Res 331: 102-106, 1996. PMID: 8895625. DOI: 10.1097/00003086-199610000-00014

11 Swanson KE, Stocks GW, Warren PD, Hazel MR and Janssen HF: Does axial limb rotation affect the alignment measurements in deformed limbs? Clin Orthop Relat Res 371: 246-252, 2000. PMID: 10693572. DOI: 10.1097/00003086-200002000-00029

12 Lemaire P, Pioletti DP, Meyer FM, Meuli R, Dörfl J and Leyvraz PF: Tibial component positioning in total knee arthroplasty: Bone coverage and extensor apparatus alignment. Knee Surgery, Sport Traumatol Arthrosc 5: 251-257, 1997. PMID: 9430576. DOI: $10.1007 / \mathrm{s} 001670050059$

13 Hunt MA, Fowler PJ, Birmingham TB, Jenkyn TR and Giffin JR: Foot rotational effects on radiographic measures of lower limb alignment. Can J Surg 49: 401-406, 2006. PMID: 17234068

14 Suter T, Zanetti M, Schmid M and Romero J: Reproducibility of measurement of femoral component rotation after total knee arthroplasty using computer tomography. J Arthroplasty 21: 744748, 2006. PMID: 16877163. DOI: 10.1016/j.arth.2005.09.012

15 Chalmers BP, Sculco PK, Fehring KA, Taunton MJ and Trousdale RT: Fluoroscopically assisted radiographs improve sensitivity of detecting loose tibial implants in revision total knee arthroplasty. J Arthroplasty 32: 570-574, 2017. PMID: 27665244. DOI: 10.1016/j.arth.2016.08.005
16 Kniesel B, Konstantinidis L, Hirschmüller A, Südkamp N and Helwig P: Digital templating in total knee and hip replacement: An analysis of planning accuracy. Int Orthop 38: 733-739, 2014. PMID: 24162155. DOI: 10.1007/s00264-013-2157-1

17 Fehring TK and McAvoy G: Fluoroscopic evaluation of the painful total knee arthroplasty. Clin Orthop Relat Res 331: 226233, 1996. PMID: 8895643. DOI: 10.1097/00003086-1996100 00-00032

18 Vyskocil P, Gerber C and Bamert P: Radiolucent lines and component stability in knee arthroplasty. Standard versus fluoroscopically-assisted radiographs. J Bone Joint Surg $\mathrm{Br} 81$ : 24-26, 1999. PMID: 10067995. DOI: 10.1302/0301-620x.81b1. 9213

19 Perka N, Kopf S and Hommel H: A whole leg radiograph is not necessary for postoperative determination of the mechanical leg axis after total knee arthroplasty. Arch Orthop Trauma Surg, 2019. PMID: 31414170. DOI: 10.1007/s00402-019-03256-x

Received May 19, 2020

Revised June 6, 2020

Accepted June 9, 2020 